\title{
Surveillance des expositions en laboratoire aux agents pathogènes humains et aux toxines, Canada 2019
}

\author{
Amanda Lien ${ }^{1}$, Christine Abalos ${ }^{1}$, Nicole Atchessi ${ }^{1}$, Rojiemiahd Edjoc ${ }^{1 \star}$, Marianne Heisz ${ }^{1}$
}

\section{Résumé}

Contexte : La Loi sur les agents pathogènes humains et les toxines et le Règlement sur les agents pathogènes humains et les toxines exigent que les incidents de laboratoire soient signalés au système de surveillance : déclaration des incidents en laboratoire au Canada (DILC) de l'Agence de la santé publique du Canada. L'objectif du présent rapport est de décrire les incidents de laboratoire concernant des expositions survenues au Canada en 2019 et les personnes affectées par ces incidents.

Méthodes : Les incidents survenus en laboratoire en 2019 dans des laboratoires canadiens autorisés ont été analysés. Le taux d'incidents d'exposition a été calculé et des statistiques descriptives ont été effectuées. Les incidents d'exposition ont été analysés selon le secteur, la cause fondamentale, l'activité, le type d'occurrence et le type de pathogène/toxine. Les personnes affectées ont été analysées selon l'éducation, la voie d'exposition, le secteur, le rôle et l'expérience en laboratoire.

Résultats : Soixante cas d'exposition touchant 86 personnes ont été signalés au DILC en 2019. Le taux d'exposition annuel était de six incidents pour 100 permis en vigueur. La plupart des cas d'exposition concernaient des activités de microbiologie ( $n=39 ; 65 \%)$ ou ont été signalés par le secteur universitaire $(n=22 ; 37 \%)$. Le secteur de la santé publique a enregistré la plus forte proportion d'incidents d'exposition alors que le secteur privé en a enregistré la plus faible proportion. L'erreur de procédure $(n=18 ; 23 \%)$ était le type d'incident le plus cité. Plus d'un tiers des personnes exposées possédaient entre 0 et 5 ans d'expérience en laboratoire $(n=32 ; 37 \%$ ) et étaient des techniciens ou des technologues du milieu hospitalier ( $n=31 ; 36 \%)$. L'inhalation était la voie d'exposition la plus courante $(n=53 ; 62 \%)$. L'interaction humaine ( $n=35 ; 24 \%$ ) était la cause fondamentale la plus citée.

Conclusion : Les incidents en laboratoire ont été moins nombreux en 2019 qu'en 2018. Le type d'incident le plus fréquent était l'erreur de procédure, tandis que les questions d'interaction humaine étaient la cause fondamentale la plus citée. La plupart des personnes exposées étaient des techniciens ou des technologues du milieu hospitalier.
Cette oeuvre est mise à la disposition selon les termes de la licence internationale Creative Commons Attribution 4.0

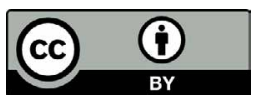

\section{Affiliation}

${ }^{1}$ Centre de la biosûreté, Agence de la santé publique du Canada, Ottawa, ON

\section{*Correspondance : \\ rojiemiahd.edjoc@canada.ca}

Citation proposée : Lien A, Abalos C, Atchessi N, Edjoc R, Heisz M. Surveillance des expositions en laboratoire aux agents pathogènes humains et aux toxines, Canada 2019. Relevé des maladies transmissibles au Canada 2020;46(9):329-36. https://doi.org/10.14745/ccdr.v46i09a07f

Mots-clés : expositions en laboratoire, incidents de laboratoire, infections contractées en laboratoire, agents pathogènes humains et toxines, surveillance, déclaration des incidents en laboratoire au Canada, Centre de biosûreté

\section{Introduction}

Lorsqu'on travaille en laboratoire avec des agents pathogènes humains et des toxines (PHT), il y a un risque inhérent d'exposition délibérée ou accidentelle. Les incidents d'expositions doivent être signalés dans des délais raisonnables sont essentiels pour atténuer le risque d'éventuelles éclosions et permettre une intervention rapide. Au cours des dernières années, ce risque pour la biosécurité humaine et les infections contractées en laboratoire (ICL) par l'exposition a conduit le 
Canada à mettre en place l'un des premiers systèmes nationaux complets de surveillance pour la déclaration obligatoire des incidents en laboratoire concernant des PHT. Au cours des années, le nombre d'ICL soupçonnées ou confirmées signalées a varié. En 2016, quatre incidents ont conduit à une ICL soupçonnée ou confirmée, et l'année suivante, sur les six ICL signalées, cinq ont été soupçonnées et une a été confirmée. En 2018, le nombre d'ICL confirmées est resté le même que celui de l'année précédente (1-3). Contrairement au système de déclaration obligatoire utilisé au Canada, la majorité des déclarations portant sur des ICL dans d'autres pays, y compris le Royaume-Uni et les États-Unis, sont faites volontairement ou sont capturées au moyen d'enquêtes (4-6).

Le Centre de la biosûreté de l'Agence de la santé publique du Canada (l'Agence) a pour mandat de protéger le public contre les risques posés par les PHT. II surveille les activités menées conformément à la Loi sur les agents pathogènes humains et les toxines (LAPHT) et au Règlement sur les agents pathogènes humains et les toxines (RAPHT). En réponse à ces exigences, le système de surveillance de déclaration des incidents en laboratoire au Canada (DILC) a été mis sur pied en décembre 2015. Sauf exception, les installations qui manipulent des PHT doivent obtenir un permis pour mener leurs activités contrôlées. Un seul permis peut couvrir plusieurs zones de confinement, mais ne couvre pas plusieurs groupes de risque (GR). Lorsqu'il s'inscrit pour obtenir un permis, chaque établissement s'identifie comme faisant partie du secteur universitaire, hospitalier, privé/commercial, de la santé publique ou d'un autre secteur gouvernemental.

Conformément à la LAPHT $(7,8)$, les PHT peuvent être classés en trois groupes de risque. La majorité des travaux effectués dans les laboratoires sous réglementation fédérale portent sur des agents pathogènes du GR2 qui présentent un risque mineur pour la santé publique, mais un risque modéré pour les personnes. Les agents pathogènes du GR3 présentent un risque élevé pour les personnes, mais un risque faible pour la santé publique. Les agents pathogènes du GR4 présentent les risques les plus élevés tant pour les personnes que pour la collectivité. Les agents biologiques à cote de sécurité élevée (ABCSE) au-dessus d'une quantité seuil peuvent présenter un risque pour la sécurité nationale du Canada. En dehors de la portée de la LAPHT, il y a les agents pathogènes du GR1, qui ne sont pas réglementés au Canada et qui présentent le risque le plus faible. Le fait de travailler avec des agents pathogènes et des toxines dans leur milieu naturel peut également présenter des risques lors d'incidents concernant des expositions et des ICL. Bien que la déclaration d'incidents concernant ces agents pathogènes et toxines ne soit pas obligatoire, l'Agence encourage la déclaration volontaire et continue de répondre à cette source de risque.
Conformément à la LAPHT, les installations autorisées qui travaillent avec des PHT du GR2 ou plus doivent signaler sans délai tout incident en laboratoire à l'Agence. Les quatre types d'incidents de laboratoire à signaler au système de surveillance de DILC sont les suivants :

- Expositions et ICL

- Possession, production ou libération involontaire d'un PHT

- PHT manquant, perdu ou volé, y compris un ABCSE non reçu dans les 24 heures suivant l'arrivée prévue

- Changements dans le confinement biologique

Le rapport initial du titulaire de permis à l'Agence à la suite d'un incident indique les dates clés, la cause de l'exposition, les personnes touchées et les PHT en cause. Un rapport de suivi est attendu dans les 15 jours suivant la première déclaration pour les incidents d'ABCSE, ou dans les 30 jours pour les autres expositions ou les ICL. Les rapports de suivi permettent $d$ 'identifier les tendances et de réduire le risque $d$ 'incidents futurs en fournissant des renseignements sur les résultats de l'enquête, le traitement et la surveillance des personnes touchées, les causes fondamentales et les mesures correctives à prendre après l'incident.

Le rapport annuel de 2019 marque la quatrième année du programme. Comme les années précédentes, le présent rapport a pour objectif de décrire la répartition des incidents en laboratoire, en mettant l'accent sur les données des expositions et les ICL. De plus, il vise à comparer les incidents d'exposition à ceux des années précédentes, à décrire les expositions en laboratoire par secteur, PHT, type d'occurrence, activité, nombre de personnes exposées (rôle principal, éducation, années d'expérience en laboratoire et voie d'exposition) et les causes fondamentales.

\section{Méthodes}

\section{Source de données}

Les rapports de déclaration et de suivi des incidents de laboratoire sont transmis par l'interface externe du Portail de biosûreté de DILC, et ces renseignements sont saisis par son système interne de gestion des relations avec la clientèle. Pour le présent rapport, les incidents de laboratoire survenus du $1^{\text {er }}$ janvier au 31 décembre 2019 ont été extraits du système de gestion des relations avec la clientèle. Les incidents qui n'avaient pas de date d'occurrence connue étaient également inclus s'ils étaient signalés au cours de cette période. Les données des rapports de suivi les plus récents ont été utilisées pour l'analyse, tandis que les données des rapports initiaux ont été utilisées lorsque les rapports de suivi ou les données correspondantes n'étaient pas présents à la date d'extraction des données, le 11 février 2020. Les données extraites ont été nettoyées en inspectant et en enquêtant sur les valeurs aberrantes et en supprimant les entrées en double. 
Dans le cadre de la LAPHT et du RAPHT, un incident d'exposition a été défini comme un incident de laboratoire qui aurait pu entraîner une intoxication/infection ou avoir entraîné une $\mathrm{ICL}$ soupçonnée ou confirmée $(7,8)$. Un incident sans exposition a trait à la possession, à la production ou à la libération involontaire d'un agent pathogène ou d'une toxine, d'un agent pathogène ou d'une toxine disparus, perdus ou volés ou d'un agent biologique à cote de sécurité élevée qui n'a pas été reçu dans les 24 heures suivant l'arrivée prévue. Les incidents concernant des agents dans le milieu naturel ont été exclus de l'analyse parce que le signalement de ces incidents est volontaire.

\section{Analyse}

Les données des rapports dans le système de surveillance DILC ont été extraites vers Microsoft Excel 2016 pour analyse et R 3.5.1 a été utilisée pour effectuer des statistiques descriptives avec validation croisée à l'aide de SAS EG 7.1. Tous les incidents d'exposition déclarés ont d'abord été subdivisés en incidents exclus et en incidents confirmés, les ICL confirmées et soupçonnées étant incluses dans ces derniers. Les personnes touchées par des incidents confirmés ont également été subdivisées en personnes confirmées ou exclues. Parmi les incidents d'exposition confirmés, le nombre d'incidents a été analysé en fonction de paramètres obtenus à deux niveaux de déclaration. Au niveau du titulaire du permis en vigueur, on a examiné la répartition des incidents signalés par secteur, activité principale, cause fondamentale, type d'occurrence et pathogène/toxine concerné. Au niveau des personnes touchées par ces incidents, on a examiné la répartition de leur niveau d'instruction le plus élevé, les années d'expérience, la voie d'exposition, le secteur et le rôle principal Une comparaison des cas d'exposition au fil du temps entre 2016 et 2019 et une mesure du taux d'exposition pour 100 permis en vigueur en 2019 ont également été effectuées. Les permis considérés comme étant en vigueur sont ceux qui étaient en vigueur en 2019 et pour lesquels un incident a pu être signalé. La période de surveillance était d'un an et a été définie comme allant du 1er janvier au 31 décembre 2019. Le calcul du taux $d^{\prime}$ incidence de l'exposition $(R)$ a été établi à partir de principes épidémiologiques bien établis (9) et a été défini comme suit :

$R=\frac{\text { nombre d'incidents d'exposition signalés pendant la période de surveillance }}{\text { total de permis en vigueur } \times \text { période de surveillance }} \times 100$ permis en vigueur

\section{Résultats}

En 2019, 996 permis en vigueur ont été détenus dans l'ensemble du Canada, permettant I'utilisation de PHT. Par rapport à 2018 $(n=89)$, il y a eu moins d'incidents confirmés signalés $(n=60)$, mais une ICL confirmée de plus $(n=2)$ (figure 1$)$.

Le taux d'exposition a été d'environ six incidents pour 100 permis en vigueur observés en 2019. Du $1^{\text {er }}$ janvier au 31 décembre 2019, 64 incidents d'exposition, 199 incidents sans exposition et 19 autres incidents ont été signalés au moyen de la
Figure 1 : Incidents d'exposition confirmés, incidents d'infection contractée en laboratoire confirmées et soupçonnées et permis en vigueur, Canada, de 2016 à 2019

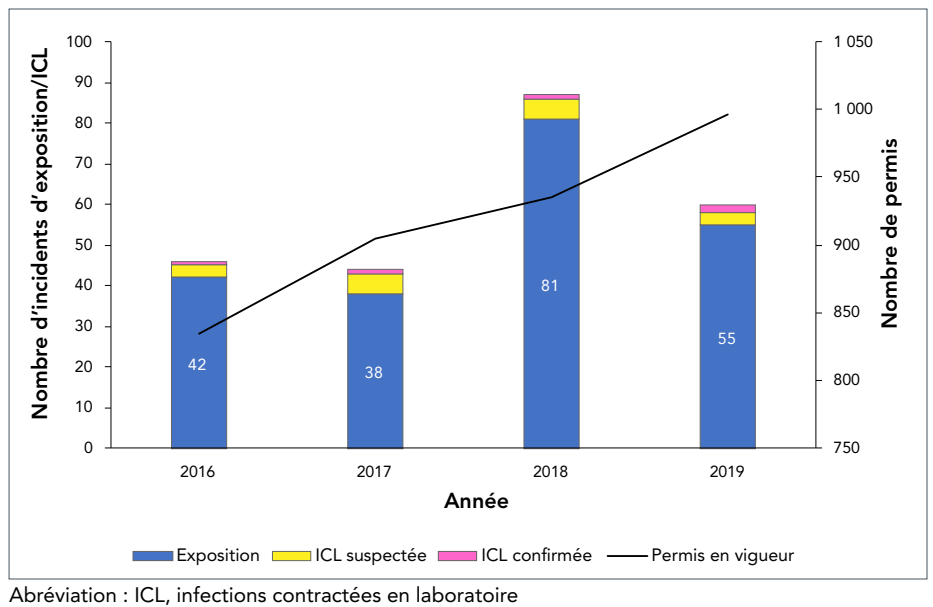

DILC (figure 2). Parmi les incidents d'exposition, quatre incidents ont été exclus de l'analyse après enquête, trois incidents ont donné lieu à des ICL soupçonnées et deux incidents ont abouti à des ICL confirmées. Au total, 99 personnes auraient été exposées dans des incidents de laboratoire, mais 13 d'entre elles ont été exclues de l'analyse après enquête pour des raisons telles que le reclassement d'un incident d'exposition comme un incident sans exposition après examen.

Figure 2 : Types d'incidents signalés à la déclaration des incidents en laboratoire au Canada et incidents d'exposition inclus dans l'analyse, Canada 2019

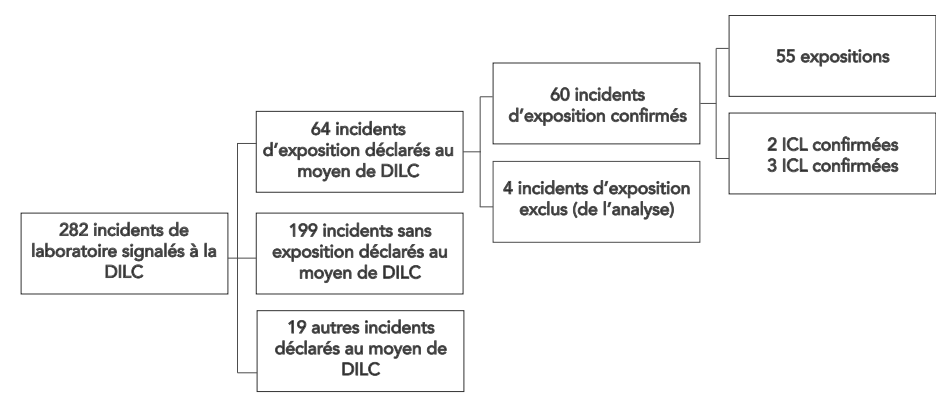

Abréviations : DILC, déclaration des incidents en laboratoire au Canada; ICL, infections contractées en laboratoire

\section{Incidents d'exposition par activité principale et par secteur}

La microbiologie était la principale activité exécutée pendant la plupart des incidents d'exposition $(n=39 ; 65,0 \%)$ suivis de la recherche in vivo sur les animaux $(n=9 ; 15,0 \%)$. Les activités les moins fréquemment citées comprennent les soins aux animaux, l'autopsie ou la nécropsie, la culture cellulaire, l'éducation ou la formation, la maintenance, les études moléculaires, la sérologie 
ou l'hématologie ou autre ( $n=12 ; 20,0 \%$ ). Les définitions des principales activités se trouvent à l'annexe $\mathbf{A}$.

La figure 3 compare le nombre d'incidents d'exposition signalés et le nombre de permis en vigueur détenus par secteur. La plupart des cas d'exposition signalés au moyen de la DILC se sont produits dans les secteurs de l'enseignement ( $n=22 ; 36,7 \%)$, hospitalier ( $n=18 ; 30,0 \%$ ) et de la santé publique ( $n=14 ; 23,3 \%$ ). De plus, le secteur de la santé publique a enregistré le plus grand nombre d'incidents d'exposition pour 100 permis détenus (40 incidents pour 100 permis en vigueur), tandis que le secteur privé a enregistré le plus faible nombre (un incident pour 100 permis en vigueur), malgré le plus grand nombre de permis en vigueur $(n=482)$.

Figure 3 : Incidents d'exposition confirmés signalés à la déclaration des incidents en laboratoire au Canada et permis en vigueur par secteur, Canada 2019

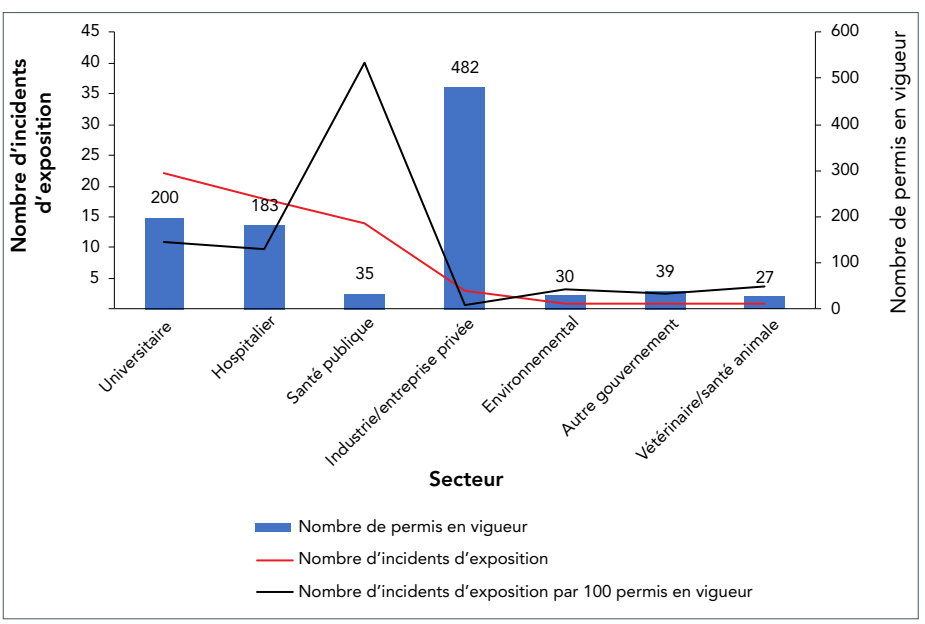

\section{Agents pathogènes humains et toxines concernés}

Salmonella était l'agent concerné dans les deux ICL confirmées qui se sont produites en 2019. Le tableau 1 montre la distribution des agents biologiques concernés dans les incidents d'exposition signalés (bactéries, champignons, parasite, virus, inconnu) par cote de sécurité (non ABCSE, ABCSE) et le groupe de risque (GR2, GR3, inconnu). Parmi les 71 agents pathogènes et toxines concernés, la plupart des cas d'exposition concernaient des agents pathogènes et des toxines non ABCSE ( $n=61 ; 85,9 \%$ ) ou se sont produits dans des laboratoires ayant un permis de GR2 ( $n=44 ; 62,0 \%$ ). Les bactéries étaient les plus concernées ( $n=45 ; 63,4 \%$ ) et les parasites étaient les moins concernés $(n=1 ; 1,4 \%)$. Les agents les plus concernés parmi les laboratoires ayant un permis de GR2 étaient Neisseria meningitidis ( $N$. meningitidis) ( $n=5 ; 7,0 \%)$, Staphylococcus aureus ( $S$. aureus) $(n=4 ; 5,6 \%$ ) et les espèces d'Escherichia ( $n=3 ; 4,2 \%$ ). L'agent le plus concerné dans les laboratoires ayant un permis de GR3 était Brucella melitensis ( $B$. melitensis) ( $n=5 ; 7,0 \%$ ).
Tableau 1 : Agents pathogènes humains ou toxines concernés dans les incidents d'exposition signalés par niveau de groupe de risque et statut de cote de sécurité élevé, Canada 2019 ( $N=71)$

\begin{tabular}{|c|c|c|c|c|c|c|}
\hline \multirow{2}{*}{$\begin{array}{c}\text { Type } \\
\text { d'agent } \\
\text { biologique } \\
\text { par groupe } \\
\text { de risque }\end{array}$} & \multicolumn{2}{|c|}{ Non ABCSE } & \multicolumn{2}{|c|}{ ABCSE } & \multicolumn{2}{|c|}{ Total } \\
\hline & $\mathbf{n}$ & $\%$ & $\mathbf{n}$ & $\%$ & $\mathbf{n}$ & $\%$ \\
\hline GR2 & 44 & 72 & 0 & 0 & 44 & 62 \\
\hline Bactéries & 32 & 52 & 0 & 0 & 32 & 45 \\
\hline $\begin{array}{l}\text { Mycète } \\
\text { (champignon) }\end{array}$ & 2 & 3 & 0 & 0 & 2 & 3 \\
\hline Parasite & 1 & 2 & 0 & 0 & 1 & 1 \\
\hline Virus & 9 & 15 & 0 & 0 & 9 & 13 \\
\hline Inconnu & 0 & 0 & 0 & 0 & 0 & 0 \\
\hline GR3 & 8 & 13 & 10 & 100 & 18 & 25 \\
\hline Bactéries & 4 & 7 & 9 & 90 & 13 & 18 \\
\hline $\begin{array}{l}\text { Mycète } \\
\text { (champignon) }\end{array}$ & 2 & 3 & 1 & 10 & 3 & 4 \\
\hline Parasite & 0 & 0 & 0 & 0 & 0 & 0 \\
\hline Virus & 2 & 3 & 0 & 0 & 2 & 3 \\
\hline Inconnu & 0 & 0 & 0 & 0 & 0 & 0 \\
\hline Inconnu & 9 & 15 & 0 & 0 & 9 & 13 \\
\hline Bactéries & 0 & 0 & 0 & 0 & 0 & 0 \\
\hline $\begin{array}{l}\text { Mycète } \\
\text { (champignon) }\end{array}$ & 0 & 0 & 0 & 0 & 0 & 0 \\
\hline Parasite & 0 & 0 & 0 & 0 & 0 & 0 \\
\hline Virus & 0 & 0 & 0 & 0 & 0 & 0 \\
\hline Inconnu & 9 & 15 & 0 & 0 & 9 & 13 \\
\hline Total & 61 & 100 & 10 & 100 & 71 & 100 \\
\hline
\end{tabular}

Remarque : Les pourcentages sont arrondis au nombre entier le plus près

\section{Types d'incidents}

La figure 4 présente les types d'incidents signalés qui sont liés à des incidents d'exposition. Les types d'incidents les plus fréquemment signalés étaient ceux liés à une procédure ( $n=18 ; 23,1 \%)$ et liés à un objet tranchant ou pointu ( $n=15 ; 19,2 \%)$.

\section{Personnes exposées}

Au total, 86 personnes ont été exposées à travers 60 incidents d'exposition confirmées signalés à la DILC. Le niveau d'instruction le plus élevé était inconnu pour sept personnes exposées. La plupart des personnes exposées possédaient un diplôme technique/professionnel ( $n=48 ; 55,8 \%$ ) ou un baccalauréat ( $n=15 ; 17,4 \%$ ). Les autres niveaux d'enseignement les plus élevés signalés comprennent le diplôme d'études secondaires ( $n=6 ; 7,0 \%)$, la maîtrise $(n=7 ; 8,1 \%)$ et le M. D. ou ou le doctorat ( $n=3 ; 3,5 \%)$. 
Figure 4 : Types d'incidents rapportés lors des incidents d'exposition signalés, Canada 2019 ( $N=78$ )

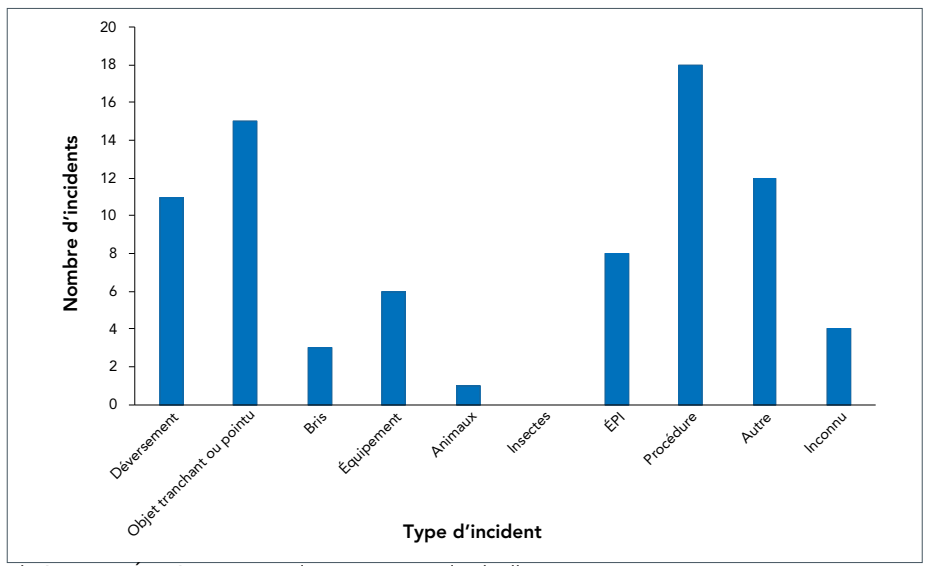

Abréviation : ÉPI, équipement de protection individuelle

Le tableau 2 présente le nombre de personnes exposées selon leur secteur et leur rôle principal. La majorité des personnes exposées appartenaient au secteur hospitalier ( $n=38 ; 44,2 \%$ ), de la santé publique $(n=22 ; 25,6 \%)$, ou universitaire ( $n=19 ; 22,1 \%$ ). La plupart des personnes exposées étaient des techniciens ou des technologues ( $n=64 ; 74,4 \%$ ) appartenant principalement aux secteurs hospitalier $(n=31)$ ou de la santé publique $(n=21)$.

La figure 5 illustre la répartition des personnes exposées selon leurs années d'expérience en laboratoire et leur rôle principal. Le nombre d'années d'expérience en laboratoire de 19 personnes exposées était inconnu. Les superviseurs et les gestionnaires $(n=2)$ avaient les années d'expérience médianes les plus élevées $(\tilde{x}=17,5)$, tandis que les étudiants $(n=13)$ avaient le plus petit nombre d'années d'expérience ( $\tilde{x}=2)$. Dans l'ensemble, la plupart des personnes exposées avaient de 0 à 5 ans d'expérience en laboratoire $(n=32 ; 37,2 \%)$.
Figure 5 : Personnes touchées par des incidents d'exposition signalés, selon le nombre d'années d'expérience $^{a}$ en laboratoire et le rôle principal ${ }^{b}$, Canada 2019 ( $N=67)$

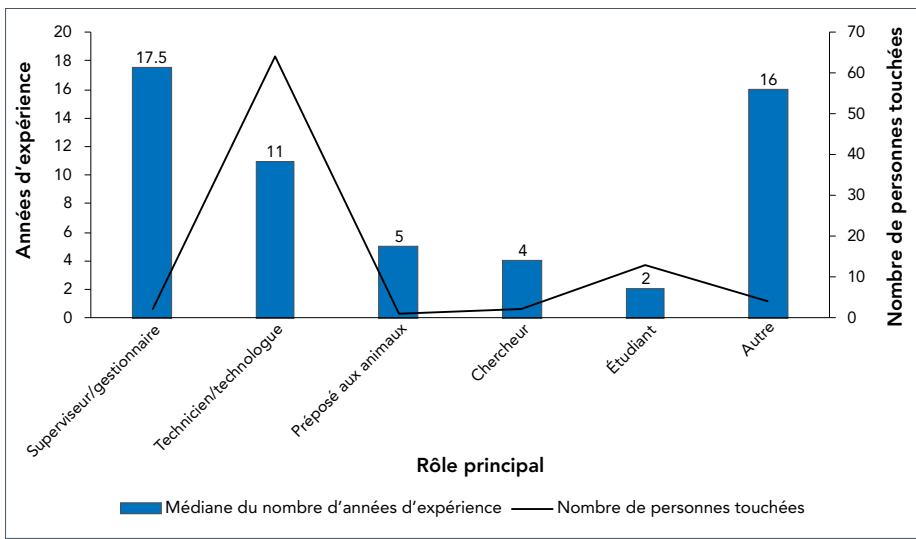

a Le niveau d'instruction le plus élevé pour 19 des 86 personnes exposées n'a pas été signalé b Les autres rôles comprennent instructeur, microbiologiste et assistant de laboratoire médical

Parmi les 86 personnes exposées, la plupart ont été exposées à des PHT par inhalation $(n=53 ; 61,6 \%)$ ou par inoculation ou injection par une aiguille ou un objet tranchant ( $n=13 ; 15,1 \%)$. Les autres voies d'exposition comprennent l'absorption par contact avec les muqueuses ou la peau, l'ingestion et l'inoculation ou l'injection par une morsure ou une égratignure.

\section{Causes fondamentales et points d'amélioration de la sécurité des laboratoires}

Au total, 144 causes fondamentales ont été identifiées dans les rapports de suivi des 60 cas d'exposition confirmés signalés. Le tableau 3 décrit les causes fondamentales et leur répartition. L'interaction humaine ( $n=35 ; 24,3 \%$ ) et les procédures opérationnelles normalisées ( $n=27 ; 18,8 \%$ ) étaient les causes

Tableau 2 : Personnes touchées par des incidents d'exposition signalés, selon le secteur et le rôle principal, Canada 2019 ( $N=86$ )

\begin{tabular}{|c|c|c|c|c|c|c|c|c|c|c|c|c|c|c|}
\hline \multirow{3}{*}{ Secteur } & \multicolumn{14}{|c|}{ Rôle principal } \\
\hline & \multicolumn{2}{|c|}{$\begin{array}{c}\text { Préposé aux } \\
\text { animaux }\end{array}$} & \multicolumn{2}{|c|}{ Chercheur } & \multicolumn{2}{|c|}{ Étudiant } & \multicolumn{2}{|c|}{$\begin{array}{l}\text { Superviseur/ } \\
\text { gestionnaire }\end{array}$} & \multicolumn{2}{|c|}{$\begin{array}{l}\text { Technicien/ } \\
\text { technologue }\end{array}$} & \multicolumn{2}{|c|}{ Autre $^{a}$} & \multicolumn{2}{|c|}{ Total } \\
\hline & $n$ & $\%$ & $\mathbf{n}$ & $\%$ & $n$ & $\%$ & $n$ & $\%$ & $n$ & $\%$ & $n$ & $\%$ & $n$ & $\%$ \\
\hline Universitaire & 1 & 100 & 2 & 100 & 9 & 69 & 1 & 50 & 5 & 8 & 1 & 25 & 19 & 22 \\
\hline Hospitalier & 0 & 0 & 0 & 0 & 4 & 31 & 0 & 0 & 31 & 48 & 3 & 75 & 38 & 44 \\
\hline $\begin{array}{l}\text { Industrie/ } \\
\text { entreprise } \\
\text { privée }\end{array}$ & 0 & 0 & 0 & 0 & 0 & 0 & 0 & 0 & 5 & 8 & 0 & 0 & 5 & 6 \\
\hline Santé publique & 0 & 0 & 0 & 0 & 0 & 0 & 1 & 50 & 21 & 33 & 0 & 0 & 22 & 26 \\
\hline $\begin{array}{l}\text { Vétérinaire/ } \\
\text { santé animale }\end{array}$ & 0 & 0 & 0 & 0 & 0 & 0 & 0 & 0 & 1 & 2 & 0 & 0 & 1 & 1 \\
\hline $\begin{array}{l}\text { Autre } \\
\text { gouvernement }\end{array}$ & 0 & 0 & 0 & 0 & 0 & 0 & 0 & 0 & 1 & 2 & 0 & 0 & 1 & 1 \\
\hline Total & 1 & 100 & 2 & 100 & 13 & 100 & 2 & 100 & 64 & 100 & 4 & 100 & 86 & 100 \\
\hline
\end{tabular}

a Les autres rôles comprennent instructeur, microbiologiste et assistant de laboratoire médical

Remarque : Les pourcentages sont arrondis au nombre entier le plus près 
fondamentales les plus fréquemment citées, suivies par la gestion ( $n=20 ; 13,9 \%$ ) et l'équipement ( $n=20 ; 13,9 \%)$.

\section{Tableau 3 : Causes fondamentales signalées dans les rapports de suivi des incidents d'exposition, Canada 2019 ( $\mathrm{N}=144)$}

\begin{tabular}{|c|c|c|c|}
\hline \multirow{2}{*}{$\begin{array}{c}\text { Cause } \\
\text { fondamentale }\end{array}$} & \multirow{2}{*}{ Exemples de point préoccupant } & \multicolumn{2}{|c|}{ Citations } \\
\hline & & $n$ & $\%$ \\
\hline \multirow[t]{2}{*}{ Communications } & $\begin{array}{l}\text { La communication n'a pas eu lieu, } \\
\text { mais aurait dû }\end{array}$ & \multirow{2}{*}{17} & \multirow{2}{*}{12} \\
\hline & $\begin{array}{l}\text { La communication n'était pas claire, } \\
\text { ambiguë, etc. }\end{array}$ & & \\
\hline \multirow[t]{3}{*}{ Équipement } & $\begin{array}{l}\text { Le contrôle de la qualité des } \\
\text { équipements doit être amélioré }\end{array}$ & \multirow{3}{*}{20} & \multirow{3}{*}{14} \\
\hline & Défaillance de l'équipement & & \\
\hline & $\begin{array}{l}\text { L'équipement n'était pas adapté aux } \\
\text { besoins }\end{array}$ & & \\
\hline \multirow[t]{2}{*}{$\begin{array}{l}\text { Interaction } \\
\text { humaine }\end{array}$} & $\begin{array}{l}\text { Une violation (prendre un raccourcis, } \\
\text { ne pas suivre la procédure correcte, } \\
\text { dévier de la procédure d'exploitation } \\
\text { normalisée) }\end{array}$ & \multirow{2}{*}{35} & \multirow{2}{*}{24} \\
\hline & $\begin{array}{l}\text { Une erreur (une erreur, un défaut } \\
\text { de concentration, ou un dérapage } \\
\text { quelconque) }\end{array}$ & & \\
\hline \multirow[t]{3}{*}{$\begin{array}{l}\text { Gestion et } \\
\text { supervision }\end{array}$} & $\begin{array}{l}\text { La supervision a besoin } \\
\text { d'amélioration }\end{array}$ & \multirow{3}{*}{20} & \multirow{3}{*}{14} \\
\hline & $\begin{array}{l}\text { Une absence de contrôle des } \\
\text { normes, politiques et procédures }\end{array}$ & & \\
\hline & $\begin{array}{l}\text { Amélioration de l'évaluation des } \\
\text { risques }\end{array}$ & & \\
\hline \multirow[t]{3}{*}{ Formation } & $\begin{array}{l}\text { Formation non en place, mais qui } \\
\text { aurait dû l'être }\end{array}$ & \multirow{3}{*}{17} & \multirow{3}{*}{12} \\
\hline & $\begin{array}{l}\text { Formation incorrecte pour la tâche } \\
\text { ou l'activité }\end{array}$ & & \\
\hline & $\begin{array}{l}\text { Le personnel n'était ni qualifié ni } \\
\text { compétent dans l'exécution de la } \\
\text { tâche }\end{array}$ & & \\
\hline \multirow[t]{3}{*}{$\begin{array}{l}\text { Procédure } \\
\text { opérationnelle } \\
\text { normalisée }\end{array}$} & $\begin{array}{l}\text { Les documents ont été suivis tels } \\
\text { qu'ils étaient écrits, mais ils n'étaient } \\
\text { pas corrects pour l'activité ou la } \\
\text { tâche }\end{array}$ & \multirow{3}{*}{27} & \multirow{3}{*}{19} \\
\hline & $\begin{array}{l}\text { Procédures non en place, mais qui } \\
\text { aurait dû l'être }\end{array}$ & & \\
\hline & $\begin{array}{l}\text { Les documents n'ont pas été } \\
\text { correctement suivis }\end{array}$ & & \\
\hline Autre & Ne s'applique pas & 8 & 5 \\
\hline
\end{tabular}

Remarque : Les pourcentages sont arrondis au nombre entier le plus près

\section{Discussion}

En 2019, 60 expositions en laboratoire aux PHT ont été signalées à la DILC, soit une diminution par rapport aux 89 expositions signalées en 2018. Sur les 60 expositions en laboratoire, cinq ont donné lieu à des ICL soupçonnées et deux d'entre elles ont été confirmées. Les agents les plus fréquemment concernés dans des incidents d'exposition étaient du GR2 ou non ABCSE. Les bactéries étaient le type d'agent le plus souvent signalé, avec $B$. melitensis, $N$. meningitidis et $S$. aureus étant le plus souvent cités. Les deux ICL confirmées ont été causées par des espèces de genre Salmonella, qui étaient aussi l'un des agents pathogènes humains les plus fréquemment responsables des ICL (10-12).

Les expositions se sont produites principalement dans les secteurs de la santé publique, universitaire et hospitalier et étaient généralement dues à des infractions à la procédure, à des objets pointus ou tranchants ou à des déversements lors d'activités de microbiologie. Quatre-vingt-six personnes, principalement des techniciens ou des technologues, ont été exposées à un PHT. Les causes fondamentales de l'exposition étaient les interactions humaines et le manque de sensibilisation ou de conformité aux procédures opérationnelles normalisées.

\section{Le secteur privé avait le plus grand nombre de permis en vigueur, mais le taux d'incident d'exposition le plus faible}

La répartition des expositions par secteur en 2019 était semblable à celle des années précédentes et les incidents se sont principalement produits dans les secteurs universitaire, hospitalier et de la santé publique (1-3). Cependant, le taux d'incidents d'exposition (9) a permis de faire une comparaison impartiale entre les secteurs en tenant compte du nombre de permis par secteur. Bien qu'ils détenaient le plus grand nombre de permis, le secteur privé avait le taux d'incident d'exposition le plus faible, alors que le secteur de la santé publique avait le taux d'incident d'exposition le plus élevé, suivi par les secteurs universitaire et hospitalier. Ces différences pourraient s'expliquer soit par une différence réelle dans le taux d'incident d'exposition d'un secteur à l'autre, soit par une différence dans la déclaration des expositions. Un taux d'incident d'exposition vraiment plus élevé dans le secteur hospitalier pourrait s'expliquer par une plus grande incertitude dans les agents pathogènes qu'ils manipulent par rapport au secteur privé, où la plupart des cas d'agents pathogènes sont déjà identifiés (e.g. dans le développement de vaccins) (11). Les facteurs qui influent sur la déclaration des incidents d'exposition selon le secteur au Canada ne sont pas bien établis et doivent être étudiés.

Le rôle et l'expérience des travailleurs de laboratoire pourraient être des facteurs importants à prendre en considération pour la prévention. En 2019, les personnes touchées qui avaient les années médianes les plus basses d'expérience en laboratoire étaient des étudiants. On s'attend à ce que le secteur universitaire soit l'endroit où les étudiants acquièrent leur première expérience dans le milieu des laboratoires et qu'ils représentent le personnel des laboratoires aux premiers stades de leur carrière. Le taux élevé d'exposition dans le secteur universitaire peut s'expliquer en partie par le lien bien établi entre le manque d'expérience et le risque accru d'erreurs (13). 
Une autre explication pourrait être la complexité de la structure organisationnelle dans les milieux universitaires, qui pourrait peut-être conduire à une méconnaissance de la responsabilité des étudiants, des chercheurs et des administrateurs. Une telle situation pourrait entraîner un non-respect des exigences de sécurité, ce qui pourrait entraîner des incidents d'exposition (14).

\section{La méconnaissance d'un incident d'exposition pourrait être une cause sous-jacente des infections acquises en laboratoire}

Comme il a été indiqué dans les rapports précédents et en parallèle avec la littérature, les agents pathogènes le plus souvent impliqués dans les incidents d'exposition en 2019 étaient les bactéries, principalement représentées par $B$. melitensis, N. meningitidis et $S$. aureus. Cependant, les deux ICL confirmées ont été causées par des espèces de genre Salmonella, qui est aussi l'un des agents pathogènes humains les plus fréquemment responsables des ICL (10-12). Dans le cas de ces deux $I C L$, aucune prophylaxie après exposition n'avait été administrée à la suite de l'incident d'exposition parce que les personnes touchées ignoraient avoir subi une exposition au moment de l'incident. L'exposition a été établie rétrospectivement après l'apparition des symptômes et du soupçon d'une ICL. L'absence de prophylaxie après exposition aurait pu être l'un des facteurs qui ont contribué à ce que l'exposition conduise à une ICL (15). L'ignorance de l'exposition aurait pu être le résultat d'interactions humaines et d'une méconnaissance des procédures opérationnelles normalisées. Ces facteurs ont souvent été identifiés comme les causes fondamentales d'expositions au cours des dernières années (1-3).

\section{Forces et limites}

La principale force de cette étude a été le processus de déclaration normalisé et obligatoire des incidents de laboratoire dans les laboratoires partout au Canada. Cela a permis d'évaluer en temps quasi réel les tendances et les possibilités d'atténuation des risques dans la prescription ou l'amélioration des mesures correctives dans les installations ayant un permis. La communication régulière avec les intervenants au moyen de publipostage électronique et de bulletins d'information permettant de déterminer les facteurs de risque potentiels. Par exemple, en 2019, l'activité principale concernée était la microbiologie. Ces renseignements pourraient être utilisés par les installations ayant un permis pour examiner les protocoles de sécurité actuels concernant cette activité afin de réduire le risque d'exposition aux travailleurs de laboratoire à l'avenir.

Le système actuel de surveillance des incidents de laboratoire ne permet pas actuellement de recueillir des renseignements comme la taille de l'effectif des travailleurs de laboratoire ou la répartition des rôles au sein des laboratoires. Par conséquent, les principales limites de ce rapport a été la précision réduite inhérente à l'utilisation de permis en vigueur comme indicateur de la taille de l'effectif des travailleurs et l'incapacité de rendre compte d'une analyse plus complète des tendances des incidents en 2019 et au fil du temps. De plus, il se peut que tous les incidents et expositions déclarés n'aient pas été signalés à la DILC. Nous continuons de nous attaquer à ce problème potentiel en menant diverses activités de conformité et de surveillance et en communiquant de façon uniforme avec les intervenants au moyen de bulletins d'information et de conseils sur la biosécurité. Nous mettons également à jour la ligne directrice sur la déclaration et le signalement afin de tenir compte du processus de signalement des agents pathogènes qui ne sont pas visés par la LAPHT/le RAPHT.

\section{Conclusion}

L'incidence annuelle d'expositions en laboratoire au Canada en 2019 a été plus faible qu'en 2018, mais plus élevée qu'en 2016 et 2017. Il demeure incertain qu'il s'agisse là d'une véritable diminution, car le programme d'ICL n'est en place que depuis 2015 et les niveaux de références sont encore en train d'être établis. L'analyse des expositions déclarées a servi à éclairer les lignes directrices pour l'amélioration continue de la biosûreté et de la biosécurité au Canada.

\section{Déclaration des auteurs}

A. L. - Méthodologie, enquête, rédaction de l'ébauche originale, révision et édition

R. E. - Conceptualisation, méthodologie, enquête, rédaction de l'ébauche originale, examen et édition, supervision

N. A. - Méthodologie, enquête, rédaction de l'ébauche originale, révision et édition

C. A. - Rédaction de l'ébauche originale, révision et édition M. H. - Révision par écrit et édition

\section{Intérêts concurrents}

Il n'y a pas de conflits d'intérêts à déclarer.

\section{Remerciements}

Nous tenons à remercier nos parties réglementées pour leur appui constant et de leur vigilance constante dans la déclaration des incidents ainsi que le personnel du Centre pour la biosûreté, pour leur expertise et leur contribution.

\section{Financement}

Ce travail a été réalisé grâce au soutien de l'Agence de la santé publique du Canada, dans le cadre de son mandat principal.

\section{Références}

1. Choucrallah D, Sarmiento L, Ettles S, Tanguay F, Heisz M, Falardeau E. Surveillance des expositions en laboratoire aux agents pathogènes humains et aux toxines: Canada 2018. Relevé des maladies transmissibles au Canada 2019;45(9):268-76. DOI 
2. Bienek A, Heisz M, Su M. Surveillance des expositions en laboratoire aux agents pathogènes humains et aux toxines au Canada en 2016. Relevé des maladies transmissibles au Canada 2017;43(11):259-68. DOI

3. Pomerleau-Normandin D, Heisz M, Tanguay F. Surveillance des expositions en laboratoire aux agents pathogènes humains et aux toxines au Canada en 2017. Relevé des maladies transmissibles au Canada 2018;44(11):377-44. DOI

4. Singh K. Laboratory-acquired infections. Clin Infect Dis 2009;49(1):142-7. DOl PubMed

5. Willemarck N, Van Vaerenbergh B, Descamps E, Brosius B, Dai Do Thi C, Leunda A, Baldo A. Laboratory- Acquired Infections in Belgium (2007-2012)_An Online Survey. Brussels (Belgium): Wetenschappelijk Instituut Volkgezondheid; 2015. https://www.researchgate.net/publication/291331348_ Laboratory-Acquired_Infections_in_Belgium_2007-2012_An_ online_survey

6. Wurtz N, Papa A, Hukic M, Di Caro A, Leparc-Goffart I, Leroy E, Landini MP, Sekeyova Z, Dumler JS,

Bădescu D, Busquets N, Calistri A, Parolin C, Palù G, Christova I, Maurin M, La Scola B, Raoult D. Survey of laboratory-acquired infections around the world in biosafety level 3 and 4 laboratories. Eur J Clin Microbiol Infect Dis 2016;35(8):1247-58. DOl PubMed

7. Gouvernement du Canada. Loi sur les agents pathogènes humains et les toxines). L.C. 2009, ch. 24. Gouvernement du Canada; 2015 (modifié 2020-04). https://lois-laws.justice. gc.ca/fra/lois/H-5.67/TexteComplet.html

8. Agence de la santé publique du Canada. Norme canadienne sur la biosécurité (NCB), Deuxième édition. Ottawa (ON) : ASPC; 2016 (modifié 2018-08). https:// www.canada.ca/fr/sante-publique/services/normes-ligne s-directrices-canadiennes-biosecurite/deuxieme-edition.html
9. Szklo M, Nieto J. Incidence rate based on person time. In: Epidemiology: Beyond the Basics. $2^{\text {nd }}$ ed.: Mississauga, ON: Jones and Bartlett; 2007. p. 57.

10. Peng $\mathrm{H}$, Bilal $\mathrm{M}$, lqbal HM. Improved Biosafety and Biosecurity Measures and/or Strategies to Tackle Laboratory-Acquired Infections and Related Risks. Int J Environ Res Public Health 2018;15(12):2697. DOI PubMed

11. Coelho AC, García Díez J. Biological Risks and Laboratory-Acquired Infections: A Reality That Cannot be Ignored in Health Biotechnology. Front Bioeng Biotechnol 2015;3:56. DOI PubMed

12. Siengsanan-Lamont J, Blacksell SD. A Review of Laboratory-Acquired Infections in the Asia-Pacific: Understanding Risk and the Need for Improved Biosafety for Veterinary and Zoonotic Diseases. Trop Med Infect Dis 2018;3(2):36. DOI PubMed

13. Plebani M. Exploring the iceberg of errors in laboratory medicine. Clin Chim Acta 2009;404(1):16-23. DOI PubMed

14. Huising R, Silbey SS. Surveillance and Regulation of Laboratory Practices. In: Felt U, Fouche R, Miller CA, Smith-Doerr L, editors. The Handbook of Science and Technology Studies. $4^{\text {th }}$ ed. Cambridge MA: The MIT Press; 2013 P.793 -822. https://mitpress.mit.edu/books/ handbook-science-and-technology-studies-fourth-edition

15. Centers for Disease Control and Prevention. Morbidity and Mortality Weekly Report (MMWR): Recommendations and Reports. 2001. Vol. 50, No RR-11, p-1-54. https://www.cdc. $\mathrm{gov} / \mathrm{mmwr} / \mathrm{PDF} / \mathrm{rr} / \mathrm{rr5011.pdf}$

\section{Annexe A}

\section{Définitions de l'activité principale}

Soins aux animaux : Activités telles que les soins quotidiens aux animaux et l'offre de traitement aux animaux

Autopsie ou nécropsie : Examens chirurgicaux post mortem à des fins telle que la détermination de la cause du décès ou l'évaluation de la maladie ou des blessures dans un but de recherche ou d'éducation

Culture cellulaire : Le processus de croissance des cellules dans des conditions contrôlées; il peut également s'agir de l'enlèvement de cellules d'un animal ou d'une plante

Éducation ou formation : Éducation ou formation des étudiants ou du personnel sur les techniques et procédures de laboratoire Recherche animale in vivo : Expérimentation avec des animaux vivants non humains

Maintenance : Entretien, réparation ou nettoyage régulier et général de l'équipement et des installations

Microbiologie : Activités concernant la manipulation, l'isolement ou l'analyse de microorganismes dans leur état viable ou infectieux Enquêtes moléculaires : Activités concernant la manipulation de matériel génétique à partir de microorganismes ou d'autres matières infectieuses en vue d'une analyse plus approfondie

Sérologie : Examen diagnostique ou étude scientifique des réactions immunologiques et des propriétés du sérum sanguin Hématologie : Étude scientifique de la physiologie du sang 